\title{
Investigation and Mechanical Modelling of Pure Molybdenum at High Strain-Rate and Temperature
}

\author{
Martina Scapin $^{1}$ (D) $\cdot$ Lorenzo Peroni $^{1} \cdot$ Federico Carra $^{1,2}$
}

Received: 26 August 2016/ Accepted: 12 October 2016/Published online: 20 October 2016

(C) Society for Experimental Mechanics, Inc 2016

\begin{abstract}
This work shows the results obtained from the investigation of the mechanical behavior of two batches of pure molybdenum specimens ( $\geq 99.97 \%$ Mo, Mo1 supplied by Plansee and Mo2 supplied by AT\&M) under static and dynamic loading conditions at different temperatures, both under tensile and compressive loading conditions. Due to its properties molybdenum has applications in several fields including nuclear. At this moment, it is a good candidate for structural material application for Beam Intercepting Devices of the Large Hadron Collider at CERN, Geneva. The experimental tests in tensile loading condition were performed on small dog-bone specimens. A series of tests at room temperature and a range of strainrates was performed in order to obtain information about the strain-rate sensitivity of the material. A series of tests at different temperatures in both static and high dynamic loading conditions was performed in order to obtain information about the thermal softening of the material. The dynamic tests were performed using the Hopkinson Bar technique, and the heating of the specimen was performed using an induction coil system. The experimental tests in compression were carried out on cylindrical specimens at room temperature and a range of strain-rates. The experimental data were analyzed via a numerical inverse method based on Finite Element numerical simulations.
\end{abstract}

Martina Scapin

martina.scapin@polito.it

1 Department of Mechanical and Aerospace Engineering, Politecnico di Torino, Corso Duca degli Abruzzi, 24, 10129 Turin, Italy

2 Engineering Department, Mechanical and Materials Engineering Group (EN-MME), 1211 Geneva 23, Switzerland
This approach allows to obtain the effective stress versus strain curves, which cannot be derived by using standard relations since instability and necking were present. Moreover, it also allows the non-uniform distribution of strain-rate and temperature inside the specimen to be accounted for. The results obtained from compression tests confirm the data obtained in tension in terms of strainhardening and strain-rate sensitivity, even if the material exhibits a tension-compression asymmetry of the behavior. The analysis of the hardening, temperature and strain-rate sensitivities reveals that a unique standard visco-plastic model could not be defined to reproduce the material strength behavior under different loading conditions, especially over wide range of variation of the variables of interest.

Keywords Refractory metal · High temperature $\cdot$ High strain-rate $\cdot$ Hopkinson bar - Tensile and compression . Nuclear applications $\cdot$ Instability $\cdot$ FE-based numerical inverse approach

\section{Introduction}

Refractory metals and their alloys found several applications in high technology fields. In particular, molybdenumbased materials have special importance in a variety of high temperature industrial and nuclear applications [1] thanks to their good combination of properties. As a matter of fact, molybdenum exhibits high strength which is maintained at high temperatures, high Young's modulus (greater than common steel), excellent thermal and electrical conductivities, high melting temperature $\left(2620{ }^{\circ} \mathrm{C}\right)$ good resistance to thermal shock and low coefficient of thermal expansion. The problems for the use of 
molybdenum are mainly related to its high temperature ductile-brittle transition (greater than $0{ }^{\circ} \mathrm{C}$ ) and oxidation at high temperature. It can work at temperatures above $1100{ }^{\circ} \mathrm{C}$ (in non-oxidizing conditions), which is higher than steels and nickel-based super-alloys.

Some of the most important fields of application for molybdenum metals and alloys are: as an alloying agent for steel, improving hardness, toughness and strength at high temperatures; as an electrode for electrically heated glass furnaces, as structural parts in aerospace, defense and nuclear energy applications; as a catalyst in the refining of petroleum; as a filament material in electronic/electrical applications; as a flame- and corrosion-resistant coatings for other metals. Furthermore, molybdenum is often chosen by many researchers as an ideal material to examine the deformation behavior of Body-Centered-Cubic (BCC) metals.

At this moment, it is used for the stiffener part for the Collimator Phase II project for the LHC machine at CERN, Geneva. Moreover, it is under study as material for Beam Intercepting Devices (BID) which have to satisfy a lot of requirements due to shock loading conditions induced by direct beam interaction $[1,2]$.

In this work the analysis of the material behavior was performed in different loading conditions, by varying both strain-rate and temperature, and a big effort was made in order to get results in case of coupled high temperature and high strain-rate conditions. In more detail, the mechanical characterization was performed at room temperature at different strain-rates in order to obtain information about the strain and strain-rate sensitivity of the material both in compression and tension. Then, two series of tensile tests at different temperatures in both static and high dynamic loading condition were performed in order to obtain information about the thermal softening of the material.

The strain-rate and temperature are variables of fundamental importance for the definition of the mechanical behavior of materials. Nevertheless, in some elastic-plastic models, the effects, coming from these two quantities, are considered to act independently, in several applications, the materials are deformed at very high speed (which implies self-heating due to adiabatic process) in high temperature condition. In this case, the stress versus strain behavior comes as the result between the effects of hardening (which is due to strain and strain-rate) and thermal softening (which is due to self-heating or environment temperature). In accordance to the fact that in several studies it was found that, since the thermal softening and strain-rate sensitivity are mutually related, not always the thermal softening obtained from quasi-static tests could be applied to predict material response under dynamic loading conditions.

The mechanical behavior of pure molybdenum has previously investigated and reported in the literature, but only few works investigating the material response over a wide range of temperature and strain-rate or both of them, especially in tension up to fracture. In a lot of works, the compressive behavior was investigated (e.g. [3-5]) from low to very high temperature conditions at different the strain-rates, ranging from quasi-static up to high strainrates. In other cases [6-8] the tensile behavior was investigated at different temperatures, but the mechanical characterization was limited to medium-low strain-rates. In some cases, e.g. [8, 9], also the effect of the irradiation was investigated and modelled. Finally, considering the results reported in [10-14], an asymmetric behavior is expected in compression and tension tests: this was a general characteristic of BCC metals and it was previously noticed on molybdenum. In a lot of the available works, the data were used to calibrate strength models (e.g. [3, 4, 9, 10, 15, 16]): different material models and techniques were applied and in some cases also compared. The choices mainly depend on the nature of the model, the available data and the final application. In a lot of works the attention was focused on Zerilli-Armstrong model, but also more physically-based and micromechanical models were identified, usually by using data interpolation techniques. Differently from analytical approaches, the inverse procedure, as that it is proposed here, can be applied for the strength model calibration also in case of necking/instability up to fracture [17-20]: a Finite Element (FE) based approach allows to take into account the effect of the geometry changes during the necking phase. The stress versus strain curve after necking starts cannot be derived using nominal relation starting from engineering data: the stress state is no longer uniform and uniaxial as a consequence of the increased triaxiality which implies the logarithmic stress differs from the Von Mises stress.

\section{Material}

The material under investigation is pure molybdenum $(\geq 99.97 \%$ Mo) provided by two different suppliers, Plansee and AT\&M and in the next section, the materials are labelled Mo1 and Mo2, respectively. The material was received in form of rods with a diameter of $6 \mathrm{~mm}$ for getting specimens for tensile tests and in form of rods with a diameter $4 \mathrm{~mm}$ for obtaining specimens for compression tests. In both the cases the material came in stress relieved condition. Due to high melting temperature, in both cases, the material was obtained via powder metallurgy. In Fig. 1, the metallographic image of the surface in the longitudinal direction is reported for Mo1. In case of Mol accordingly to the producer specifications, at room temperature in quasi-static regime, the expected value of the yield stress at $0.2 \%$ of deformation is at minimum $380 \mathrm{MPa}$ with an 


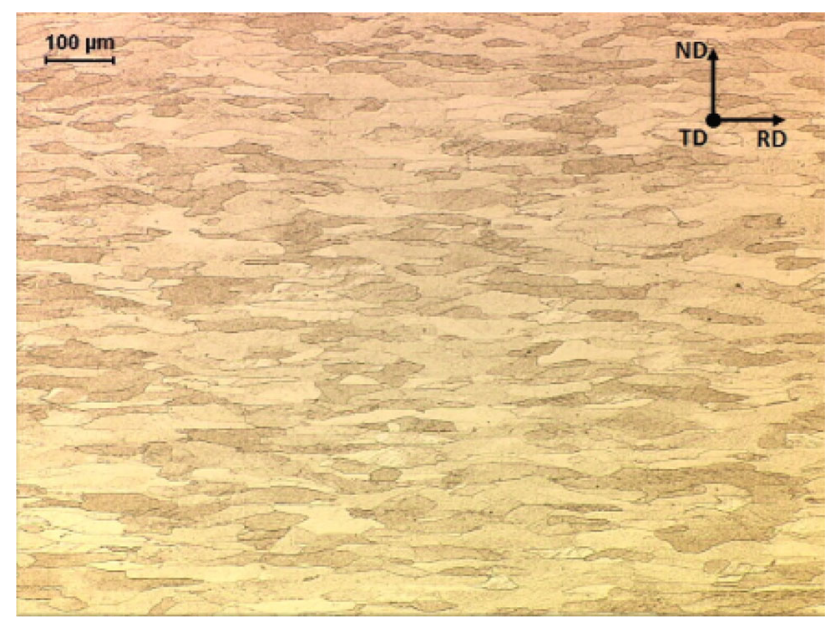

Fig. 1 Metallographic picture in the longitudinal direction

elongation greater than $15 \%$. For the material supplied by AT\&M (Mo2), the declared minimum guaranteed yield strength is $560 \mathrm{MPa}$ at room temperature with an elongation at failure greater than $5.6 \%$. Despite the producers' specifications the Mo1 strength is expected to be lower than those of Mo2, the results of the present testing campaign revealed that the behavior of the two materials is very similar.

\section{Experimental Results}

As mentioned in the previous section, the mechanical characterization was performed in both tension and compression. In tension, tests at different temperatures and strain-rates were performed, while in compression only the strain-rate sensitivity was investigated. The investigation in compression was limited since the reachable level of strain is much lower than in tension, but nevertheless increasing the strain means also to increase the friction, which is difficult to be taken into account during data elaboration. As widely discussed in [21], to perform tests in compression at high temperature, especially in dynamic regime, is more critical due to the small dimensions of the specimens. For these reasons for the data analysis the results coming from compression tests were not used.

In compression the investigated range in strain-rate was between $10^{-3}$ and $10^{3} \mathrm{~s}^{-1}$. The tests were performed on cylindrical specimens nominally with $4 \mathrm{~mm}$ of diameter and $4 \mathrm{~mm}$ of length.

In the tensile case, a dog-bone specimen was used (see Fig. 2). A series of tests at room temperature at different strain-rates was performed in order to obtain information about the strain-rate sensitivity of the material. The nominal strain-rates of the tests were $10^{-3}, 10^{-1}$ and $10^{1} \mathrm{~s}^{-1}$ (both in compression and tension). At $10^{3} \mathrm{~s}^{-1}$ in tension, the material behavior is brittle: the early failure or the failure in the threaded ends makes difficult to get reliable results. A series of tensile tests at different temperatures in static loading condition was performed in order to obtain information about the thermal softening of the material. The nominal temperatures of the tests were: $200,400,600$ and $800{ }^{\circ} \mathrm{C}$. Finally, a series of tensile tests at different temperatures in high dynamic loading condition was performed in order to obtain information about the thermal softening of the material at high strain-rate (nominally at $10^{3} \mathrm{~s}^{-1}$ ). The nominal temperatures of the tests were: 100 , 200, 300, 400, 600, 800, 90 and $1000^{\circ} \mathrm{C}$. At this loading rate, a temperature of $100{ }^{\circ} \mathrm{C}$ represents the lower temperature at which the results are reliable. Both for quasistatic and high dynamic loading conditions, the specimen was heated using an induction coil system, controlled with a feedback on the temperature measurement obtained using thermocouples directly welded on the specimen surface.

In tension and compression, the low strain-rate tests were performed on a standard electro-mechanical testing machine Zwick Z-100 (maximum load $100 \mathrm{kN}$, maximum travel speed $5 \mathrm{~mm} / \mathrm{s}$ ). The medium strain-rate tests were performed on a standard servo-hydraulic testing machine Dartec HA100 (maximum load $100 \mathrm{kN}$; maximum speed $100 \mathrm{~mm} / \mathrm{s}$ ). The high strain-rate tests were performed using Split Hopkinson Bar setups in direct configuration. A more in depth description of the testing setups can be found in [21-23]. In the tests at elevated temperature, both in quasistatic and dynamic regime, the specimen was heated using an induction coil system, controlled with a feedback on the temperature measurement obtained using thermocouples directly welded on the specimen surface (see Fig. 2).

For each testing condition at least three tests were performed in order to control the data scattering, but for the sake of clarity only the average curves are reported in Figs. 3 and 4. The estimation of the data scatter is performed by calculating the mean standard deviation for each loading condition, as reported in Table 1 for Mo1 and in Table 2 for Mo2. In general, the scatter for the Mo2 is slightly higher, but for both the materials the level of repeatability is high. In the following section, due to the limited data scatter, for each loading condition a single curve was used for the data elaboration to have the possibility to keep the correspondence between the experimental curve and post-mortem analyzed specimen. In each case, the chosen experimental curve is the closest to the mean curve.

The load applied by the testing equipment and the deformation of the specimen were recorded. Starting from these data, the average force versus stroke curves were obtained and the corresponding engineering stress versus engineering strain curves are reported in Fig. 3 for Mo1 and Fig. 4 for Mo2. 
Fig. 2 Sketch of the specimen used for tensile tests (d $=3 \mathrm{~mm}, \mathrm{~L}=5 \mathrm{~mm})$; picture of the hot specimen taken during a high speed test performed at $800{ }^{\circ} \mathrm{C}$
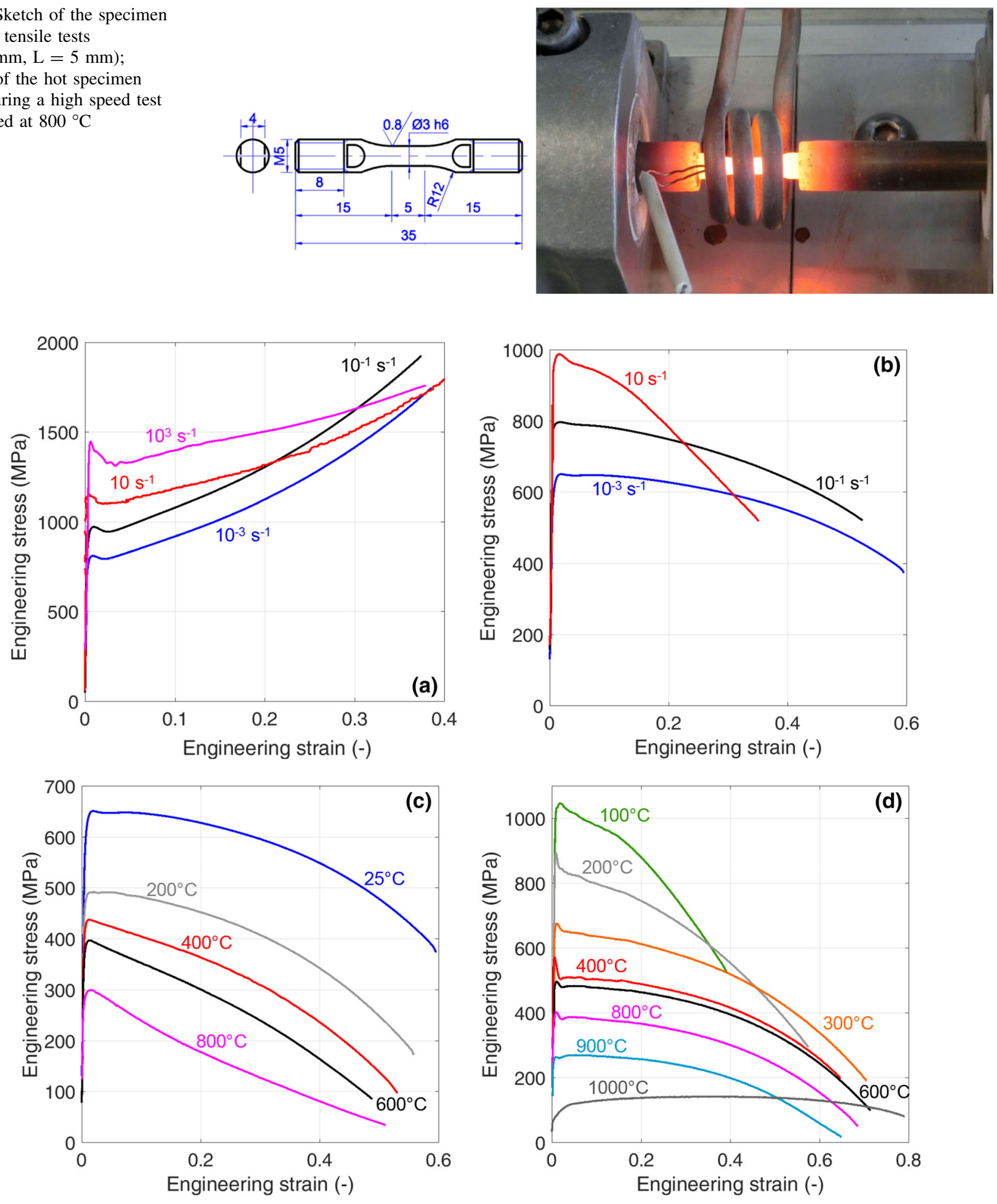

Fig. 3 Mo1-Engineering stress versus engineering strain curves obtained from the experimental test campaign (the average curve is shown for each testing condition): a compression tests at room temperature varying the strain-rate; $\mathbf{b}$ tensile tests at room

From the results it is possible to notice that, in general, both the materials exhibit a yielding instability, which is in accordance with the results find by other researchers (e.g.

temperature varying the strain-rate; $\mathbf{c}$ quasi-static tensile tests varying the temperature; $\mathbf{d}$ tensile tests in dynamic loading condition varying the temperature

$[3,7,8])$. The instability is present also in compression for Mo1, while Mo2 does not show instability in compression. By comparing the material strength in compression and 

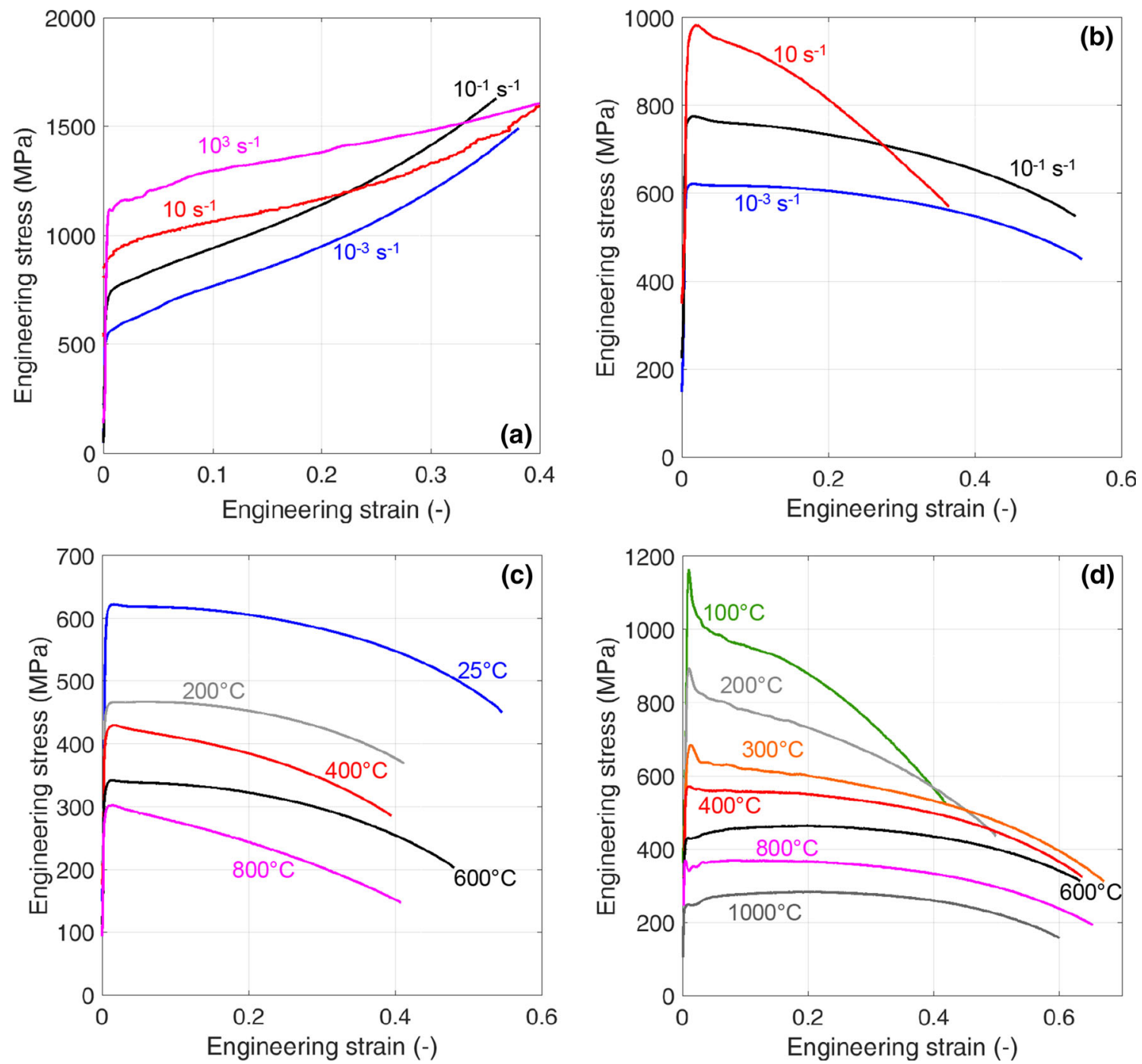

Fig. 4 Mo2-Engineering stress versus engineering strain curves obtained from the experimental test campaign (the average curve is shown for each testing condition): a compression tests at room temperature varying the strain-rate; $\mathbf{b}$ tensile tests at room

temperature varying the strain-rate; $\mathbf{c}$ quasi-static tensile tests varying the temperature; $\mathbf{d}$ tensile tests in dynamic loading condition varying the temperature

Table 1 Mo1-Data scatter evaluation in terms of standard deviation $(\mathrm{MPa})$

\begin{tabular}{lllllllllll}
\hline $\mathrm{SR}\left(\mathrm{s}^{-1}\right)$ & $\mathrm{T}\left({ }^{\circ} \mathrm{C}\right)$ & & & & & & & & & \\
\cline { 2 - 9 } & 25 compression & 25 tension & 100 & 200 & 300 & 400 & 600 & 800 & 900 & 1000 \\
\hline $10^{-3}$ & 8.3 & 6.5 & - & 7.5 & - & 9.8 & 8.7 & 26 & - & - \\
$10^{-1}$ & 11 & 10 & - & - & - & - & - & - & - & - \\
$10^{1}$ & 20 & 22 & - & - & - & - & - & - & - & - \\
$10^{3}$ & 9.4 & n.a. & 11 & 11 & 14 & 4.4 & 7.4 & 16 & 16 & 25
\end{tabular}

tension, it is possible to notice that the material strength is higher in compression. The same result was found also in [10-14]. As well known, this is the typical behavior expected for BCC metals and it was previously notice precisely on molybdenum. As widely discussed in the scientific literature, it is related to the fact that different mechanisms occur during plastic deformation and dislocation movements.

Both the materials are strongly strain-rate and temperature sensitive, as expected for a BCC material. For both the materials and both in tension and compression, the strain-hardening at low and high strain-rate is different 
Table 2 Mo2-Data scatter evaluation in terms of standard deviation (MPa)

\begin{tabular}{lllllllllll}
\hline SR $\left(\mathrm{s}^{-1}\right)$ & $\mathrm{T}\left({ }^{\circ} \mathrm{C}\right)$ & & & & & & & & \\
\cline { 2 - 9 } & 25 compression & 25 tension & 100 & 200 & 300 & 400 & 600 & 800 & 900 & 1000 \\
\hline $10^{-3}$ & 35 & 25 & - & 33 & - & 33 & 24 & 7.2 & - & - \\
$10^{-1}$ & 27 & 28 & - & - & - & - & - & - & - & - \\
$10^{1}$ & 47 & 3.5 & - & - & - & - & - & - & - & - \\
$10^{3}$ & 16 & n.a. & 6.7 & 32 & 20 & 31 & 14 & 19 & - & 8.5 \\
\hline
\end{tabular}

starting from $10^{1} \mathrm{~s}^{-1}$. In addition to this phenomenon, especially in compression, it is possible to notice a component of thermal softening at high level of strain for tests at $10^{3} \mathrm{~s}^{-1}$ : the tests show a reduction of the strength with the increase of strain due to self-heating of the specimen (the tests could be considered as adiabatic): over a certain amount of deformation, the plastic work becomes relevant as well as the heat generated inside the specimen. In this condition, the thermal softening balances and overcomes the effect of the strain-rate hardening. At high strain-rate in compression the maximum strain investigated is limited to $35 \%$ due to the length of the pulse in SHPB tests and achievable impact speed.

For both the materials, all the performed tensile tests, with the exception of few cases, exhibit the necking initiation at yielding or immediately after: the engineering stress decrease increasing strain after yielding due to the reduction in the effective resistant section. The BCC refractory metals due to the high yield stress and relatively low hardening ratio reach the instability condition, $\mathrm{d} \sigma / \mathrm{d} \varepsilon=\sigma$, for extremely low level of strain and often the whole test is in necking region independently from the size of the specimen and the type of test.

By a direct comparison between the curves obtained for the two materials, it is possible to conclude that the two materials, differently from the producers' specifications, show very similar behaviors. The yield strength obtained from quasi-static tests at different temperatures are perfectly in accordance with the data reported by Plansee [24].

Increasing the temperatures (both in quasi-static and dynamic cases), the materials strength decreases, showing a sort of plateau effect starting from $400{ }^{\circ} \mathrm{C}$, especially in case of Mo1. In the same manner, also the elongation at failure increases (this does not necessarily imply higher strain at failure). Since, for the molybdenum the recrystallization is expected to be between 900 and $1100{ }^{\circ} \mathrm{C}$, some of the tests at higher temperature show the transition behavior: in particular, the dynamic test on Mol at $1000{ }^{\circ} \mathrm{C}$ exhibits a drop in strength with respect to the test at $800{ }^{\circ} \mathrm{C}$ (for this reason the test at $900{ }^{\circ} \mathrm{C}$ was added). For Mo2 the effect of the recrystallization was not appreciable up to $1000^{\circ} \mathrm{C}$.
As the strain-rate increases both the materials harden and reduce the elongation at failure. This was also proved by the fact that it was not possible to obtain results from tests at high strain-rate and room temperature in tension: the materials were too brittle. A minimum temperature of $100{ }^{\circ} \mathrm{C}$ was sufficient for performing the dynamic test.

The material response obtained from tests at different temperatures in quasi-static conditions need to be discussed. Differently from the high strain-rate tests, in quasistatic conditions, the specimen remains hot for a long time and this may cause microstructural changes occurring during the specimen deformation. Moreover, a gradient of temperature is developed along the longitudinal direction of the specimen due to the fact that the specimen changes position with respect to the solenoid: the electromagnetic coupling changes, hence the temperature calibration is no longer valid. On the contrary, this does not happen in high strain-rate tests, in which it is possible to switch-off the heater during the test without causing a decrement in temperature (the test duration is about $600 \mu \mathrm{s}$ ). To solve the problem a symmetric testing machine should be used to maintain the specimen fix or the solenoid should be moved with the specimen to follow its motion, but even in this case the effect of the generated magnetic field in a specimen with different geometry respect to the initial condition could not be clearly predicted.

Due to the presence of the solenoid for the specimen heating, it was not possible to get the video of the test and the post-mortem analysis of each specimen is the only possibility to estimate the strain at failure. For each specimen, the post-mortem diameter $(d)$ was used to calculate the strain at fracture by the relation:

$\varepsilon_{f}=2 \ln \frac{d_{0}}{d}$

where $d_{0}$ is the initial diameter of the gage section. Due to the fact that a big portion of the specimen was deformed in a non-uniform way and the necking phase is a considerable part of the entire test, the only possibility to estimate the strain at failure was the measure of the cross section. The obtained value of strain at failure represents a valid metric under the assumptions that it is considered as the average 


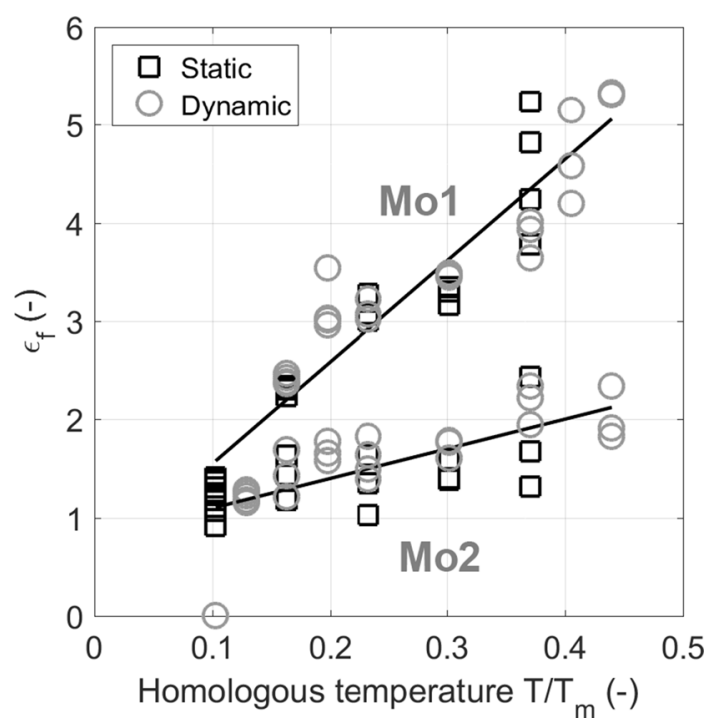

Fig. 5 Strain at failure obtained from the post-mortem measurements of the diameter; analytical fit with a linear model as a function of temperature

values over the entire section and the volume is constant (no ductile damage occurs, i.e. the ductile damage was not considered).

In Fig. 5, the calculated strains at failure vs. homologous temperature are reported: the data obtained in quasistatic and dynamic loading conditions are compared. The data were fitted by a linear relation as a function of temperature. In Fig. 6, the images of the fracture surfaces for some loading conditions are reported both for Mo1 and Mo2.

The results show that, as expected from the producers' specifications, the strain at failure of Mo1 is higher with respect to that of Mo2 at room temperature. Moreover, the difference between the two materials is amplified by increasing the temperature. For both the materials, the strain at failure is substantially insensible to the strain-rate: the increase for the increment in strain-rate is inside the data dispersion.

\section{Data Analysis}

As previously mentioned and widely discussed in the scientific literature (e.g. in [20]), in case of tensile test, the true stress versus strain curve after necking starts cannot be derived using nominal relation starting from engineering data: the stress state is no longer uniform and uniaxial as a consequence of the increased triaxiality which implies the true stress differs from the Von Mises stress. This means that any further elaboration of the experimental data requires to get the equivalent stress versus strain curves for each loading condition. With this aim, the experimental data should be processed via a numerical inverse method based on FE simulations in order to obtain the equivalent (Von Mises) stress versus effective plastic strain of the material. The main objective of an inverse optimization method is the determination of the selected set of unknown
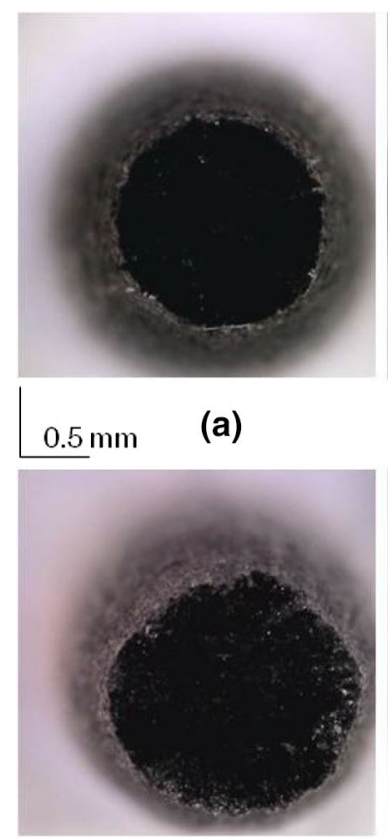

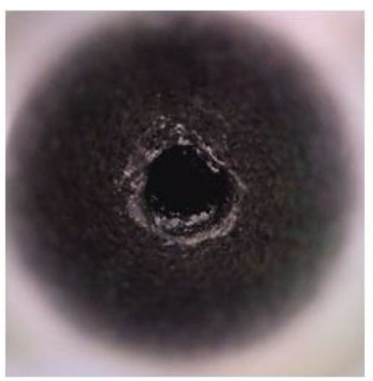

(b)

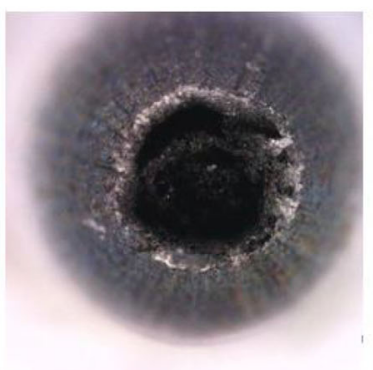

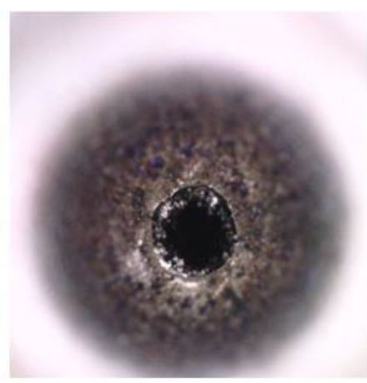

(c)

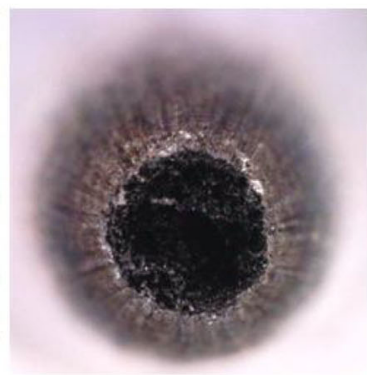

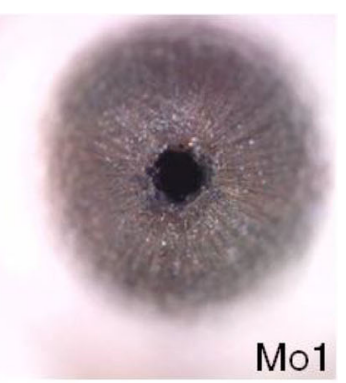

(d)

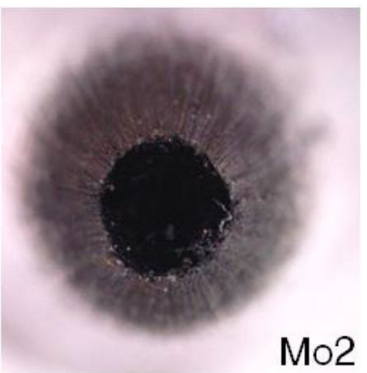

Fig. 6 Frontal images of the fracture surface taken with a digital microscope: a room temperature at $10^{-3} \mathrm{~s}^{-1} ; \mathbf{b} 400{ }^{\circ} \mathrm{C}$ at $10^{-3} \mathrm{~s}^{-1} ; \mathbf{c} 400{ }^{\circ} \mathrm{C}$ at $10^{3} \mathrm{~s}^{-1} ; \mathbf{d} 800{ }^{\circ} \mathrm{C}$ at $10^{3} \mathrm{~s}^{-1}$ 
parameters of the chosen constitutive relation in a numerical model: starting from a trial point, the unknown parameters are estimated iteratively by comparing experimentally measured with numerically computed quantities. In standard approach the comparison is made in terms of macroscopic material response, such as force versus stroke curve. Another important consideration is that for materials like BCC ones, which exhibit a strong dependency on strain-rate and temperature, a classical approach, in which these quantities are included in the hardening component (valid approach for example for FCC materials) cannot be adopted.

By taking into account these considerations, one wants to achieve a valid description of the paths followed by strain, stress, strain-rate and temperature over the entire deformation process for each loading condition. At this stage two possibilities can be followed: multi-cases or single-case optimizations. The first method implies to identify a unique strength model by taking into account at the same time the results coming from all the loading conditions. In this way the final result is a set of parameters for a strength model which is able to average, in the best manner as possible, the material behavior over very wide ranges in strain, strain-rate and temperature. By following this approach, the description of each loading condition should not be so accurate, while the global behavior could be acceptable. On the other hand, the single-case method can be used to describe in a more precise manner what is the real evolution of all the variables of interest in a single test for a more limited range of variation of the variables. The problem in this case could be related to the uniqueness of the solution. One reason could be that there are a lot of parameters to be determined and often different parameters can produce the same effects on the material response. Moreover, it is not guaranteed that the set of parameters, which is able to reproduce the macroscopic material response (e.g. force vs. stroke curve) with a good level of accuracy, it is also able to reproduce the correct deformed shape of the necking zone. This is a general result, but it becomes more relevant if, as in this case, the material shows early instability or necking, which are responsible of the localization of deformation. From this, the idea to add to the optimization process the information about the shape of the necking zone, which contains all the necessary data: the entire path of strain, which in turn determines strain-rate (since it is the deformation rate) and temperature (in case of adiabatic increment of temperature). By summarizing, the followed procedure is a single-case optimization with two objectives: the macroscopic material response and the final shape of the necking area. The objective of each optimization was the minimization of the total Normalized Mean Squared Error (NMSE).

The problem to face with at this point is the choice of the strength material model. By looking at the results reported in Figs. 3 and 4, it appears evident that the materials behavior cannot be correctly and completely described either by a simple multiplicative model such as Johnson-Cook (J-C) [25] or a more suitable for BCC additive and coupled material model such as the ZerilliArmstrong (Z-A) one [26]. The $\mathrm{J}-\mathrm{C}$ model was chosen because it is simple to manage in an optimization procedure, with a limited number of parameters. In support of this choice there is the fact that for the single-case method, the range of variation of the involved variables (in particular strain-rate and temperature) is limited, hence also a simple model such as $\mathrm{J}-\mathrm{C}$ could be adequate for the description of a local point of view.

\section{Results}

The main goal of each optimization process was the identification of the best set of parameters of the $\mathrm{J}-\mathrm{C}$ model able to simultaneously minimize the distance between experimental and computed force versus stroke curves and match the specimen profile in the necking area. The flow stress is defined by the $\mathrm{J}-\mathrm{C}$ model as follows:

$\sigma=\left(A+B \varepsilon^{n}\right)\left(1+C \ln \frac{\dot{\varepsilon}}{\dot{\varepsilon}_{0}}\right)\left(1-\left(\frac{T-T_{r}}{T_{m}-T_{r}}\right)^{m}\right)$

in which the parameters to be determined are $A, B$ and $n$ for the hardening part, $C$ for the strain-rate sensitivity and $m$ for the thermal softening description. For low and medium strain-rate the self-heating was not taken into account since the tests are isothermal, so the thermal part was neglected. For all the loading conditions the final stroke of the FE simulation corresponds to the maximum stroke applied to the specimen during the test and consequently to the time at which the shape of the necking area is controlled.

In each case, the optimization of the parameters was performed with a dedicated algorithm included in the software LS-OPT, that manages the parameter variation strategy, runs the numerical simulation, performed in LSDYNA, analyses the results and extracts the optimum set of parameters. The starting values were obtained from literature [3] and in order to accelerate convergence a domain reduction strategy is adopted to reduce the size of the subregion of the feasible points. During a particular iteration, the subregion is used to bound the position of new points. All the simulations were performed with an explicit integration method on a 2D axisymmetric model with subintegrated elements (one integration point for each element). A 2D-remeshing algorithm was applied in order to control the element dimension and get reliable results even if high value of plastic strain was reached during the necking phase. In order to reduce the computational time for low strain-rate tests the deformation rate was increased 
with respect to the experimental conditions, under the assumption that the strain-rate distribution is supposed to be the same. The boundary conditions were applied to the specimen ends in terms of velocity profiles.

The results of the single-case optimizations are reported in Figs. 7 and 8 for Mo1 and Mo2 respectively in terms of comparison between experimental and computed force versus stroke curves. As it is possible to appreciate, in the most cases the comparison is good and this means the models are able to reproduce the corresponding experimental data over the entire range of strain.

In Figs. 9 and 10, a more qualitative comparison is reported: the computed shape of the necking area is overlapped to the experimental image of the specimen. Obviously in the portion of the specimen close to the fracture surface, the differences could be more relevant: the shape of this part strongly depends on ductile damage that the specimen experienced before fracture and which was not considered in the FE calculations.

In Figs. 11 and 12, the obtained equivalent stress (Von Mises) versus equivalent plastic strain curves are reported for all the loading conditions. Each curve is interrupted at the maximum level of equivalent plastic strain computed at the end of the corresponding numerical simulation. This approach allows to take into account the increase in the triaxiality due to the new deformed geometry, which is
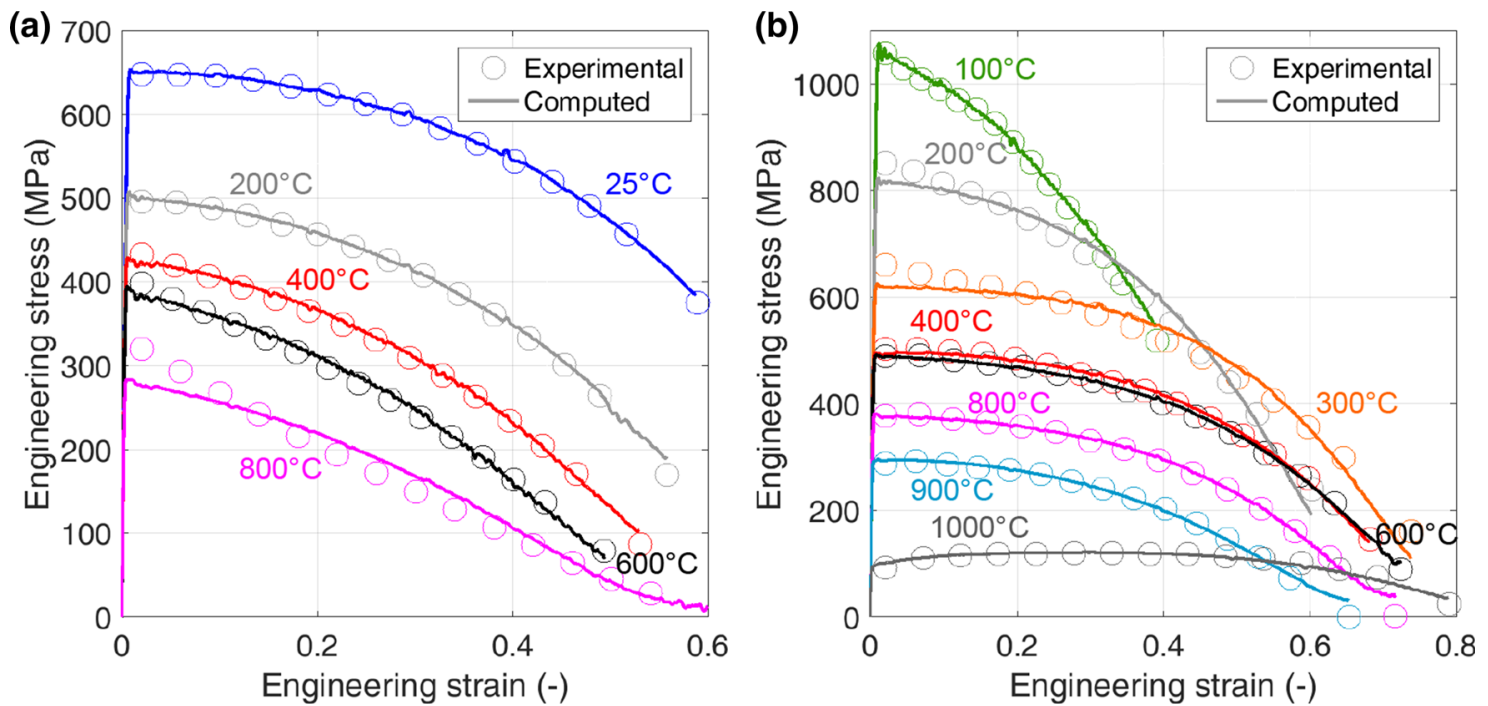

Fig. 7 Mo1-Comparison between experimental and computed results in terms of engineering stress versus engineering strain curves: a quasistatic tensile tests varying the temperature; $\mathbf{b}$ tensile tests in dynamic loading condition varying the temperature
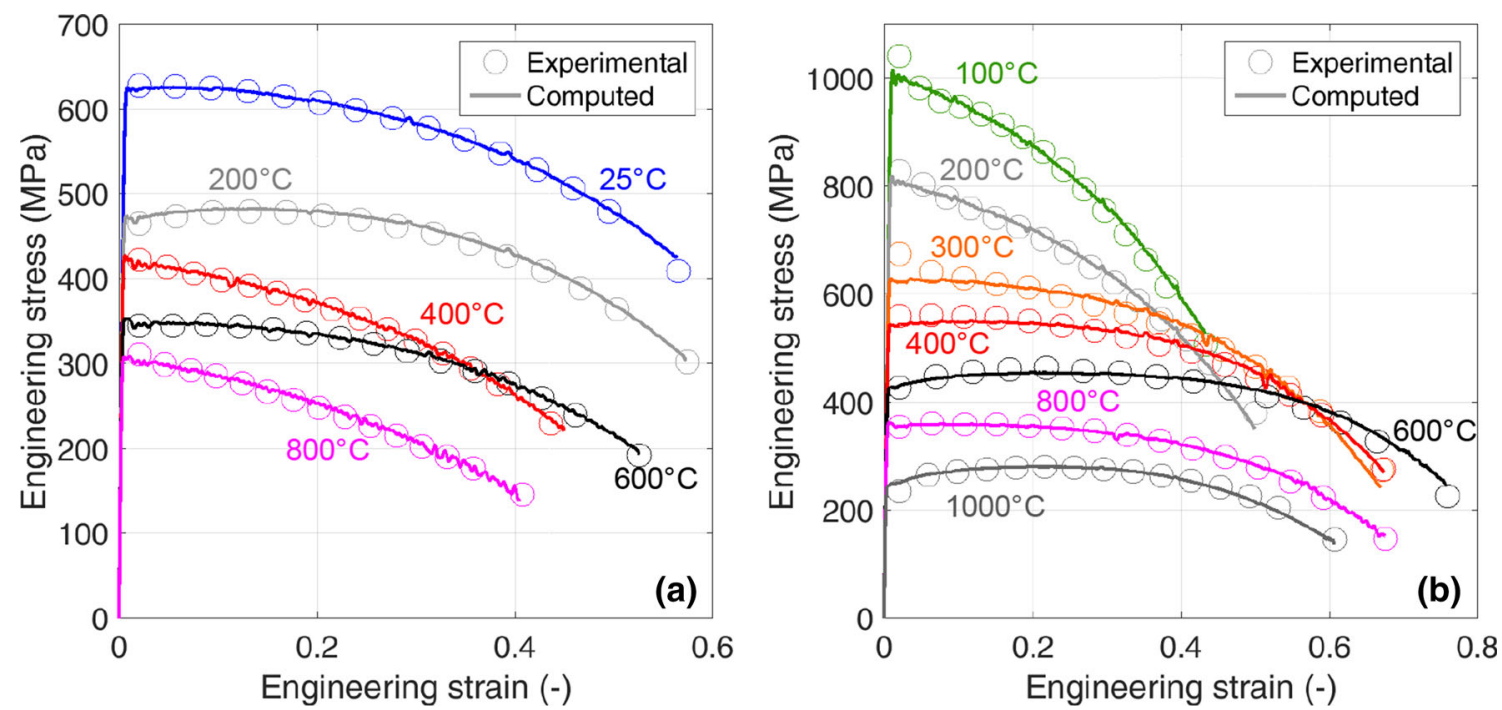

Fig. 8 Mo2-Comparison between experimental and computed results in terms of engineering stress versus engineering strain curves: a quasistatic tensile tests varying the temperature; $\mathbf{b}$ tensile tests in dynamic loading condition varying the temperature 

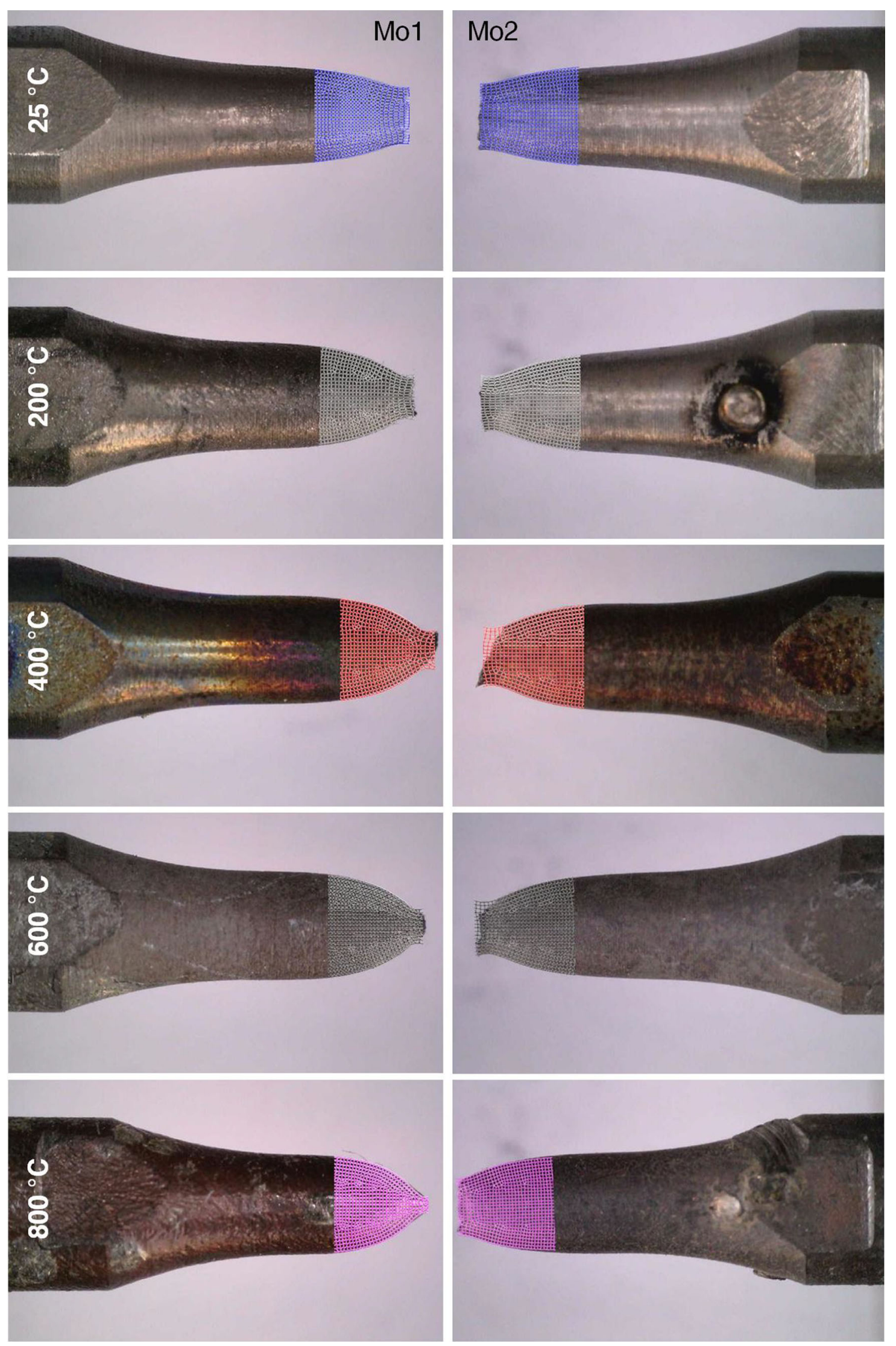

Fig. 9 Experimental and computed results in terms of final shape of the necking area for Mo1 and Mo2 for quasi-static loading condition 

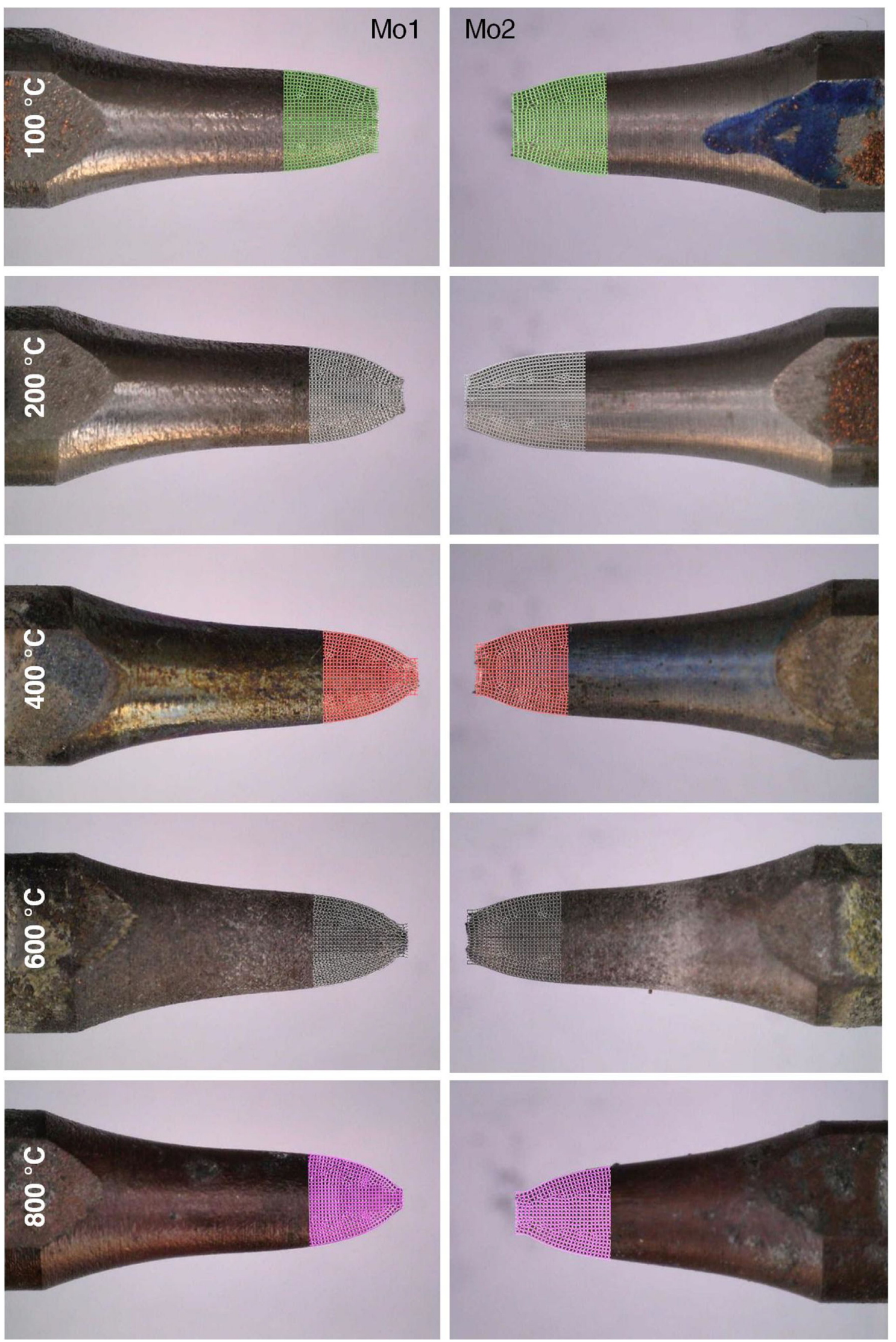

Fig. 10 Experimental and computed results in terms of final shape of the necking area for Mo1 and Mo2 for high strain-rate tests 

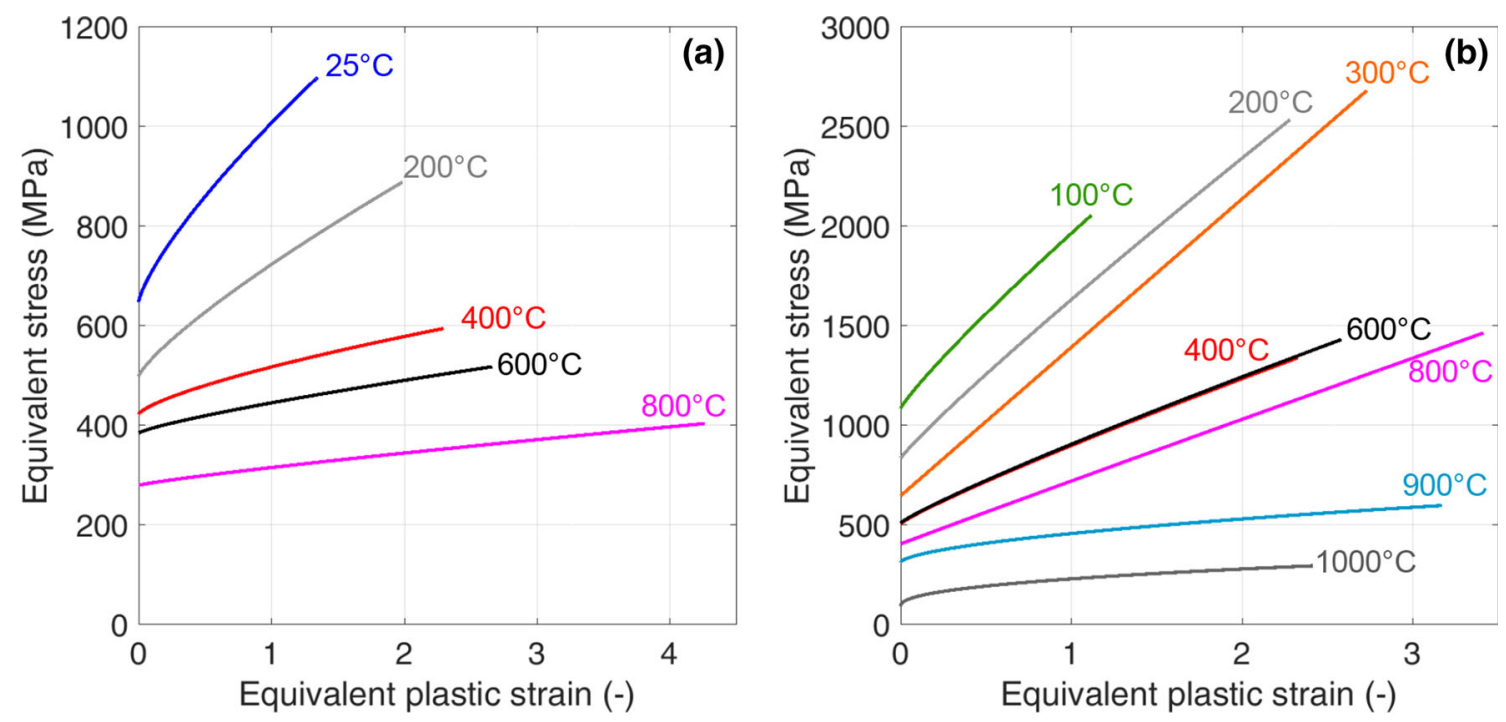

Fig. 11 Mo1-Optimized Johnson-Cook models: a quasi-static tensile tests varying the temperature; b tensile tests in dynamic loading condition varying the temperature
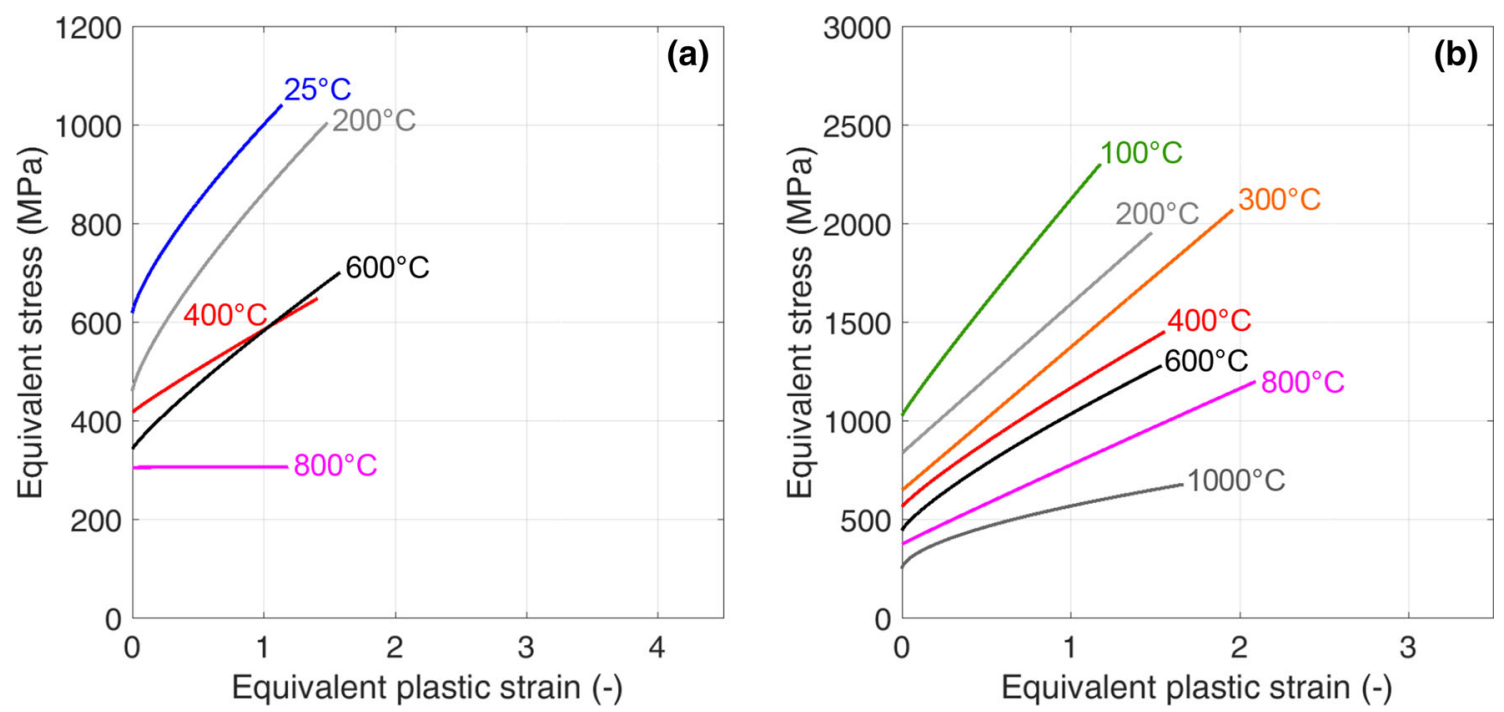

Fig. 12 Mo2-Optimized Johnson-Cook models: a quasi-static tensile tests varying the temperature; b tensile tests in dynamic loading condition varying the temperature

automatically computed by the FE model. As previously mentioned, the behavior of each specimen point follows a path on a surface defined as a function of strain, strain-rate and temperature (in the FE models all these contributions were taken into account). In order to simplify the understanding, in Figs. 11 and 12, all the curves are shown as a function of effective plastic strain for the initial levels of strain-rate and the initial temperature (isothermal flow stress): by considering strain-rate and temperature as constant, each point of the specimen terminates to a different value of plastic strain, which corresponds to the maximum value obtained in the middle of the minimum cross section.
As it is possible to notice, the maximum levels of strain are very high especially for Mo1. For the most part of the cases, the obtained flow stress is linearly dependent on the strain, and this is strictly related to the presence of early instability.

Figures 13 and 14 shows the summary of the results for Mo1 and Mo2. In Fig. 13, the equivalent stress at a plastic strain of $0.2 \%$ is reported as a function of temperature. For isothermal cases, the results are single points in correspondence to the test temperature. In case of dynamic test, the results depend on temperature in accordance to the $\mathrm{J}-\mathrm{C}$ relation, between the test temperature (starting point) and 

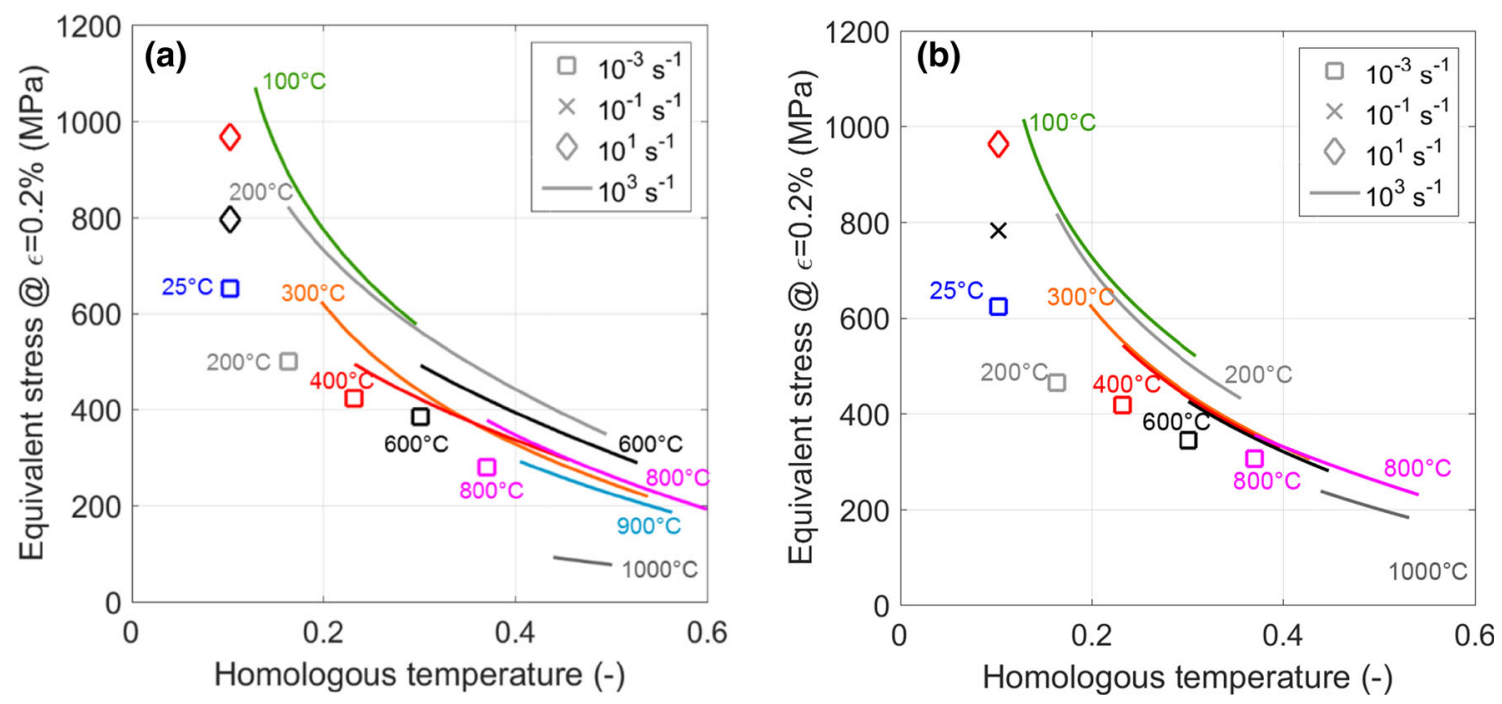

Fig. 13 Summary of the temperature sensitivity for Mo1 (a) and Mo2 (b)
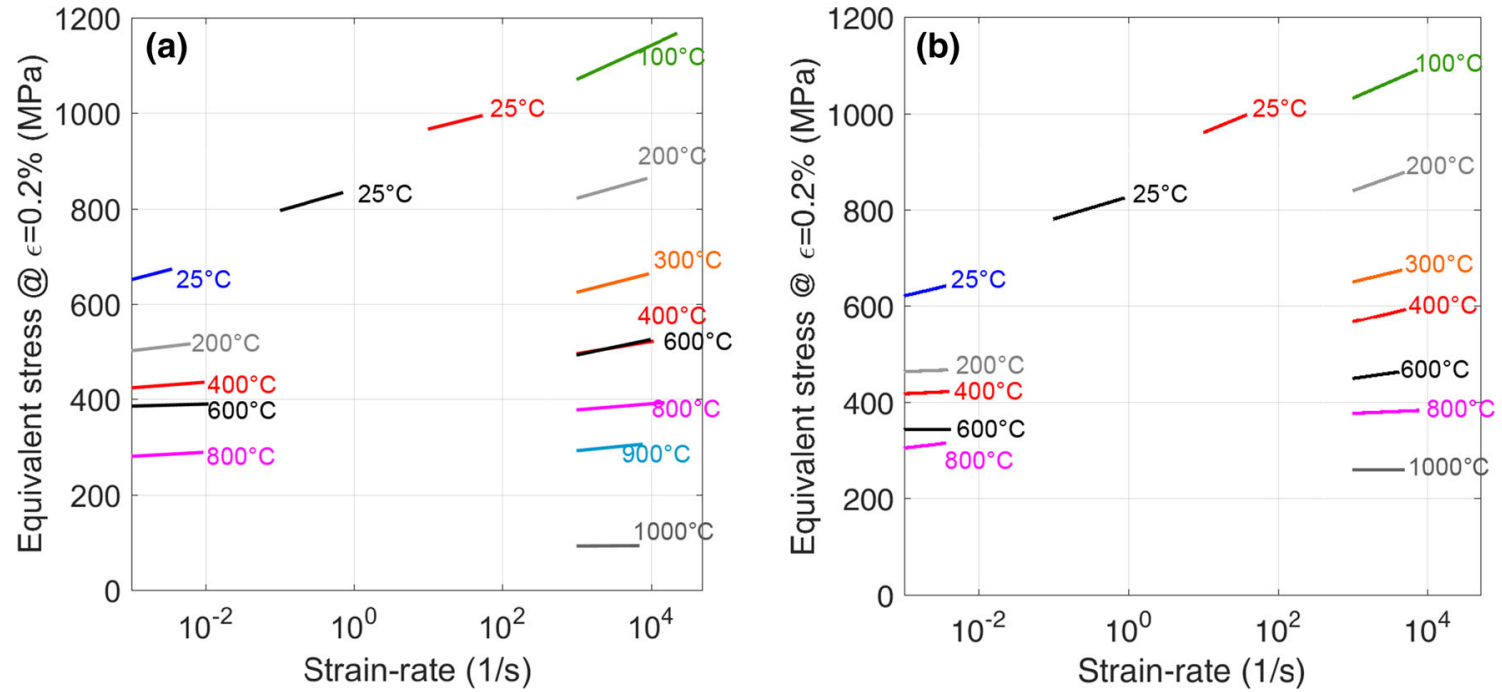

Fig. 14 Summary of the strain-rate sensitivity for Mo1 (a) and Mo2 (b)

maximum temperature, which was computed by FE simulation. Similarly, in Fig. 14 the equivalent stress is reported as a function of strain-rate between the nominal value (starting point) and the maximum computed strainrate, for all the loading conditions.

\section{Discussion of the Results}

The results obtained for Mo1 and Mo2 have to be interpreted as local results: this means that some inconsistencies can be observed by looking all the results together and this does not invalidate any results. As a matter of fact, by looking all the obtained results they appear congruent. Moreover, they predict some expected behaviors: the decrease of both the hardening rate and the strain-rate sensitivity by increasing the temperature, the increase of the strain-rate sensitivity by increasing the strain-rate itself, a different thermal softening for quasi-static and dynamic regimes with a different thermal softening responses at various temperatures but with similar trends (high strainrate).

In comparison with some of the results which can be found in the scientific literature there are some differences. For example, by comparing the results with those obtained in [3], the shape of the hardening curve is different, but a valid explanation could be the extremely smaller range of strain investigated in [3]. On the other hand, the analysis of the strain at failure reveals that for this test campaign, the values of strain at failure are very high and this is confirmed by considering both the post-mortem as well as the 

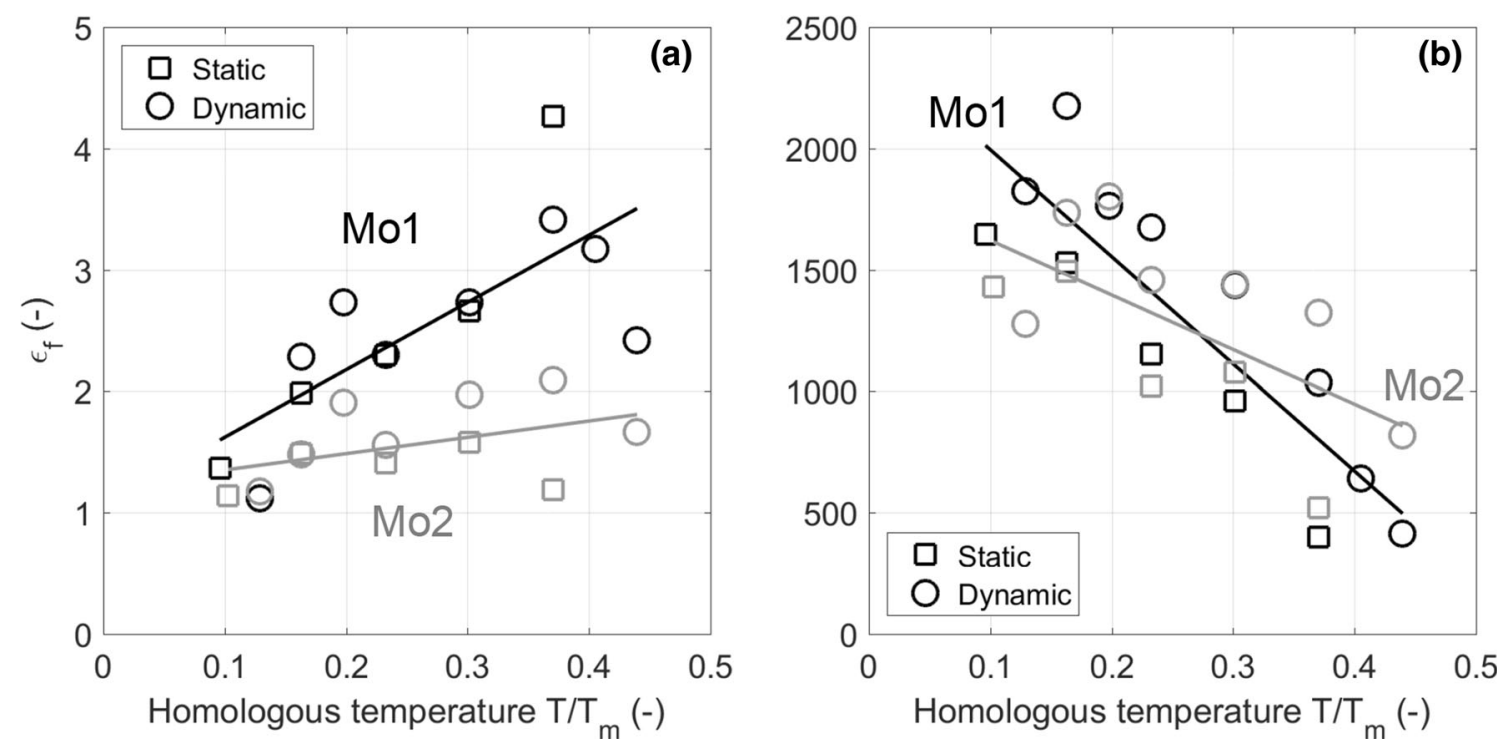

Fig. 15 Computed equivalent plastic strain at failure (a) and maximum principal stress at failure (b); analytical fit with linear models as a function of temperature
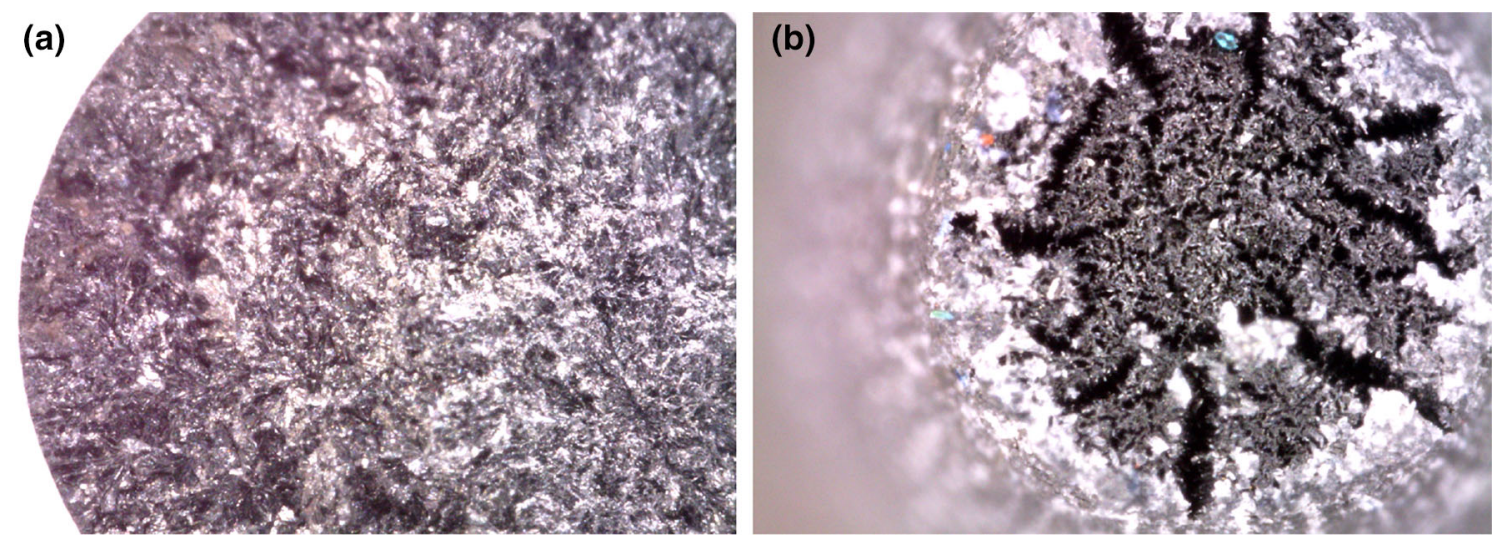

Fig. 16 Optical microscope images of the fracture surface $(150 \times)$ : dynamic test at room temperature (the results in terms of force-stroke curve are not reliable) with brittle fracture (a); quasi-static test at room temperature with ductile damage (b)

computed values. In Fig. 15, the computed values of effective plastic strain and maximum principal stress at failure are reported as a function of the homologous temperature. As it is possible to notice, for both the materials, at low temperatures, the maximum principal stress at failure is more or less constant and can be estimated (the results are quite scattered) equal to $1900 \mathrm{MPa}$ for Mo1 and $1600 \mathrm{MPa}$ for Mo2. If in this range of temperatures, the yield stress is close to this limit in stress, the material fails before yielding (DBTT) or at low percentage of plastic strain and the failure is brittle (see Fig. 16a). By increasing the temperature (or decreasing the strain-rate), the mechanisms of failure are dependent on temperature and the material is subjected to ductile damage (see Fig. 16b): for both the material, even if the maximum principal stress at failure is decreasing, the strain at failure increases. In particular, Mo1 shows higher strain at failure with lower maximum principal stress at failure and this can be explained by different flow stress curves.

At this stage, as final elaboration, an analytical fit of all the data, expressed in terms of equivalent stress versus equivalent plastic strain, could be performed with a unique strength model. It is important to underline that just having a look to the diagrams of Figs. 11, 12, 13 and 14, it appears evident that neither Zerilli-Armstrong or Johnson-Cook models could be used. As a matter of fact, the requirements for a satisfactory data fit should imply a model able to: increase the yielding with strain-rate and decrease it with temperature but with different relations in temperature and strain-rate, respectively; modify the hardening rate as a function of temperature and strain-rate; be able to model plateau effect as a function of temperature and modification material strength over a certain temperature to model recrystallization. 
A solution in order to obtain reliable results could be to focus the attention on limited ranges in temperature and strain-rate.

\section{Conclusions}

In this work a testing campaign was performed in order to investigate the mechanical behavior of two batches of pure molybdenum specimens (Mo1, supplied by Plansee and Mo2, supplied by AT\&M) under static and dynamic loading conditions at different temperatures both under tensile and compressive loading conditions. In tension, the strain-rate and the thermal softening were investigated; while in compression only the influence of strain-rate was studied. Moreover, a post-mortem analysis was performed to get information about the failure behavior, which was modelled with a linear model as a function of temperature.

The results showed that, in general, both the materials exhibit a yielding instability in tension, but only one material showed it in compression. Both the materials exhibit an asymmetric behavior in compression and tension, with higher material strength in compression probably due to a different mechanism of deformation. Both the materials are strain-rate and temperature sensitive with a coupling effect between the two quantities. As expected, recrystallization occurs above $900{ }^{\circ} \mathrm{C}$ for one material, while the other one showed a higher stability at high temperature. The thermal softening due to heat conversion of plastic work was confirmed in compression at high strain-rate, but seemed to not have effect at high temperature in tension.

The experimental data were processed via a numerical inverse method based on Finite Element (FE) numerical simulations to get the true stress versus true strain curve, since standard relations could not be applied in tension due to instability and necking. In comparison with a standard approach, it was found to be extremely necessary to include strain-rate and temperature effects in the numerical model. Moreover, in order to impose the consistency between the macroscopic response of the material (expressed in terms of force vs. stroke curve) and the necking, it was necessary to perform multi-objectives optimizations. This kind of procedure was implemented independently for each loading condition.

In conclusion, if the aim is to get a unique material model for the description of material behavior, it will be necessary that it satisfy a certain number of conditions. The model should be able to: reproduce a different behavior between compression and tension; be able to both scaled and translated the material strength as a function of temperature and strain-rate; coupled in temperature and strainrate in order to take into account a different thermal softening in different strain-rate loading conditions; model the dependence on temperature with a non-linear relation, in order to be able to represent different forms of change in the trend. From all these considerations, it can be concluded that it is not possible to find a unique strength model among which are usually used, able to reproduce with a good level of accuracy the complete behavior of material.

Acknowledgments This work was performed within the WP 11 of the FP7 European Project EUCARD-2. The authors are grateful to the financial support of the European Commission by means of the EUCARD project and to the Mechanical and Materials Engineering Group of the Engineering Department (EN-MME) of CERN (Geneva) for providing the two grades of molybdenum for this study.

\section{References}

1. Bertarelli A et al (2013) An experiment to test advanced materials impacted by intense proton pulses at CERN HiRadMat facility. Nucl Instrum Meth B 308:88-99

2. Dallocchio A, Bertarelli A, Arnau Izquierdo G, Artoos K (2009) Advanced materials for future phase II LHC collimators, Proceedings of PAC09, Vancouver

3. Nemat-Nasser S, Guo W, Liu M (1999) Experimentally-based micromechanical modeling of dynamic response of molybdenum. Scripta Mater 40(7):859-872

4. Chen C, Yin H, Humail IS, Wang Y, Xuanhui Q (2007) A comparative study of a back propagation artificial neural network and a Zerilli-Armstrong model for pure molybdenum during hot deformation. Int J Refract Metal Hard Mater 25:411-416

5. Xiao M, Li F, Xie H, Wang Y (2012) Characterization of strengthening mechanism and hot deformation behavior of powder metallurgy molybdenum. Mater Des 34:112-119

6. Hollang L, Brunner D, Seeger A (2001) Work hardening and flow stress of ultrapure molybdenum single crystals. Mater Sci Eng A 319-321:233-236

7. Meng B, Wan M, Xiangdong W, Zhou Y, Chang C (2014) Constitutive modeling for high-temperature tensile deformation behavior of pure molybdenum considering strain effects. Int $\mathbf{J}$ Refract Metals Hard Mater 45:41-47

8. Li M, Eldrup M, Byun TS, Hashimoto N, Snead LL, Zinkle SJ (2008) Low temperature neutron irradiation effects on microstructure and tensile properties of molybdenum. J Nucl Mater 376:11-28

9. Krishna S, De S (2011) A temperature and rate-dependent micromechanical model of molybdenum under neutron irradiation. Mech Mater 43:99-110

10. Kleiser GJ, Revil-Baudard B, Cazacu O, Pasiliao CL (2015) Plastic deformation of polycrystalline molybdenum: experimental data and macroscopic model accounting for its anisotropy and tension-compression asymmetry. Int $\mathrm{J}$ Solids Struct 75-76:287-298

11. Kleiser GJ, Revil-Baudard B, Pasiliao CL (2016) High strain-rate plastic deformation of molybdenum: experimental investigation, constitutive modeling and validation using impact tests. Int $\mathbf{J}$ Impact Eng 96:116-128

12. Kim JY, Jang D, Greer JR (2011) Crystallographic orientation and size dependence of tension-compression asymmetry in molybdenum nano-pillars. Int J Plast 28(1):46-52

13. Wang ZQ, Beyerlein IJ (2011) An atomistically-informed dislocation dynamics model for the plastic anisotropy and tensioncompression asymmetry of BCC metals. Int J Plast 27:1471-1484 
14. Healy C, Ackland G (2014) Molecular dynamics simulations of compression-tension asymmetry in plasticity of Fe nanopillars. Acta Mater 70:105-112

15. Cheng J, Nemat-Nasser S, Guo W (2001) A unified constitutive model for strain-rate and temperature dependent behavior of molybdenum. Mech Mater 33:603-616

16. Skoro GP, Bennett JRJ, Edgecock TR, Booth TN (2012) Yield strength of molybdenum, tantalum and tungsten at high strain rates and very high temperatures. J Nucl Mater 426:45-51

17. Noble JP, Goldthorpe BD, Church P, Harding J (1999) The use of the Hopkinson bar to validate constitutive relations at high rates of strain. J Mech Phys Solids 44:1187-1206

18. Zhang ZL, Hauge M, Odegard J, Taulow C (1999) Determining material true stress-strain curve from tensile specimens with rectangular cross!section. Int J Solids Struct 36:3497-3516

19. Rodriguez-Martinez JA, Rittel D, Zaera R, Osovski S (2013) Finite element analysis of AISI 304 steel sheets subjected to dynamic tension: the effects of martensitic transformation and plastic strain development on flow localization. Int J Impact Eng $54: 206-216$
20. Mirone G (2013) The dynamic effect of necking in Hopkinson bar tension tests. Mech Mater 58:84-96

21. Scapin M (2015) Mechanical characterization and modeling of the heavy tungsten alloy IT180. Int J Refract Metal Hard Mater 50:258-268

22. Scapin M, Peroni L, Fichera C (2014) Mechanical behavior of glidcop Al-15 at high temperature and strain rate. J Mater Eng Perform 23:1641-1650

23. Scapin M, Peroni L, Fichera C (2014) Investigation of dynamic behavior of copper at high temperature. Mater High Temp 31(2):131-140

24. https://www.plansee.com/en/materials/molybdenum.html

25. Johnson GR, Cook WA (1983) A constitutive model and data for metals subjected to large strains, high strain rates and high temperatures. Proceeding of 7 th International Symposium on Ballistics; 541-547

26. Armstrong RW, Zerilli FJ (1998) Dislocation mechanics based analysis of material dynamic behavior. J Phys IV 49:529-534 